\title{
Begrenzte Evidenz für chinesische Arzneitherapie bei COVID-19: Systematisches Review und Metaanalyse
}

\section{Originalpublikation}

Xiong XJ, Wang $P Q$, Su KL et al (2020) Chinese herbal medicine for coronavirus disease 2019: A systematic review and meta-analysis. Pharmacol Res. https://doi.org/10.1016/j.phrs.2020.105056. Online ahead of print.

\begin{abstract}
Currently, coronavirus disease 2019 (COVID-19), which can lead to severe respiratory failure and death, is now a global pandemic with no specific anti-viral drugs or vaccines. However, it is worth noting that traditional Chinese medicine (TCM), especially Chinese herbal medicine (CHM), has been widely applied in mainland China since outbreak, bringing new hope for the prevention and control of COVID-19. A comprehensive literature searching was conducted in 7 electronic databases from their inception up to June 21, 2020 to evaluate the efficacy and safety of CHM for COVID-19. Eighteen randomized controlled trials (RCTs) involving 2275 patients were enrolled. Most of CHMs were originated from classical Chinese herbal formulas. Liquoric Root (Gancao, Radix Glycyrrhizae), Baical Skullcap Root (Huangqin, Radix Scutellariae Baicalensis), Pinellia Rhizome (Banxia, Rhizoma Pinelliae Tematae), Forsythia Fruit (Lianqiao, Fructus Forsythiae Suspensae), and Bitter Apricot Seed (Kuxingren, Semen Armeniacae Amarum) were most frequently used Chinese herbs. The most commonly used dosage formulation was decoction. Our meta-analyses found that comparing CHM group and conventional western medicine group, CHM group has improvements in several clinical parameters including lung CT, clinical cure rate, ranging from mild to critical cases, length of hospital stay, total score of clinical symptoms, fever reduction time, symptom score of fever, number of cough reduction cases, symptom score of cough, number of fatigue reduction cases, symptom score of fatigue, disappearing time of fatigue, TCM syndrome, viral nucleic acid testing, and inflammatory biomarkers (C-reactive protein). Besides, no severe adverse effects were identified by CHM. CHM, especially classical Chinese herbal formulas, could be used as potential candidates for COVID-19 in this battle.
\end{abstract}

Deutsche Zeitschrift für Akupunktur 2020 • 63 (4): 221-222

https://doi.org/10.1007/s42212-020-00313-0

Online publiziert: 12 . Oktober 2020

(c) Springer Medizin Verlag GmbH, ein Teil von Springer Nature 2020

\section{Zusammenfassung der Studie}

Die chinesischen Autoren führten ein Review samt Metaanalyse zu randomisierten kontrollierten Studien durch, die die Wirksamkeit und Sicherheit einer chinesischen Arzneitherapie bei COVID-19 zum Gegenstand hatten. Einschlusskriterium waren Studien, die in der Behandlungsgruppe chinesische Arzneitherapie - ggf. plus westliche Medizin - und in der Kontrollgruppe westliche Medizin (in identischer Form) - ggf. plus TCM-Placebo - verwendeten.

Hauptzielgröße war eine Verbesserung des Thorax-CT-Befundes, Nebenzielgrößen waren: die Sterberate, die klinische Heilungsrate, die Veränderung hinsichtlich eines milden oder kritischen Verlaufs, die Krankenhausverweildauer, die Besserung von Symptomen (Symptomgesamtscore, Fieber, Husten, Fatigue), die Virus-RNA-Konversionsrate sowie Entzündungswerte einschließlich Leukozyten, Neutrophile, Lymphozyten und CRP.

Sieben internationale bzw. chinesische Datenbanken wurden systematisch durchsucht.

Die methodische Qualität wurde unabhängig von 2 Reviewern beurteilt.

Nach Ausschluss von Duplikaten fanden sich 4393 Arbeiten, von denen aber nur 18 die Einschlusskriterien erfüllten. Alle stammten aus China und waren bis auf eine (in Englisch) in chinesischer Sprache veröffentlicht. Die eingeschlossenen Studien umfassten 2275 Patienten, die Probandenzahl der einzelnen Studien lag zwischen 20 und 517. Alle verglichen eine kombinierte Therapie aus westlicher und chinesischer Medizin mit alleiniger westlicher Medizin. Ein TCM-Placebo wurde nicht eingesetzt. Die Behandlungsdauer variierte zwischen 5 und 15 Tagen. Der Hauptzielparameter Thorax-CT wurde in 13 Studien als Kriterium verwendet, die Mortalität in 4 Studien. Nebenwirkungen wurden in 10 Studien dokumentiert.

Die methodische Qualität wurde nach den 8 Kriterien des Cochrane-Handbuchs beurteilt. Die Einhaltung der weitaus meisten Kriterien blieb in den Studien unklar, überwiegend konnte nur ein Qualitätskriterium definitiv erfüllt werden, 5 Studien erfüllten 2 und eine Studie 3 der 8 Kriterien.

Aus der chinesischen Medizin kamen 31 verschiedene Kombinationsmittel zur Anwendung, denen im Wesentlichen klassische Rezepturen zugrunde lagen. Die Hälfte davon wurde in Form von Dekokten verabreicht, 4 Mittel als Injektionspräparate. Am häufigsten war Lianhua-Qingwen-Granulat vertreten. (Die DZA hatte in den Ausgaben 2 und 3/2020 über das Mittel berichtet.) Die Mittel enthielten 100 verschiedene Einzeldrogen. Die häufigsten waren (in absteigender Reigenfolge): Glycyrrhizae Radix 
et Rhizoma (gan cao), Scutellariae Radix (huang qin), Pinelliae Rhizoma (ban xia), Forsythiae Fructus (lian qiao) und Armeniacae Semen amarum ( $k$ u xing ren). Ephedrae Herba (ma huang) wurde in 2 Dritteln der Studien mit eingesetzt.

Im Hauptzielparameter, dem Thorax-CT, kam es zu Veränderungen, die in der TCM-plus-Gruppe der 13 Studien, die diesen Parameter erhoben, hochsignifikant günstiger ausfielen als in der westlichen Medizingruppe $(n=1402$; RR $=1,23,95 \% \mathrm{CI}$ : $1,15-1,32, p<0,00001)$. Die Sterblichkeitsrate fiel in den 4 Studien, die darüber Angaben machten, nicht signifikant verschieden aus $(n=463$; RR $=0,34,95 \% \mathrm{CI}: 0,05-2,18, p=0,26)$. Alle klinischen Nebenkriterien (die Heilungsrate, die Veränderung hinsichtlich eines milden oder kritischen Verlaufs, des Symptomgesamtscores, von Fieber, Husten und Fatigue) zeigten signifikante Unterschiede zugunsten der TCM-plus-Gruppe.

An objektiven Ergebnissen zeigte sich in Studien, die Laborparameter einschlossen, in der TCM-plus-Gruppe eine höhere Konversionsrate bzgl. Virus-RNA und eine vermehrte CRP-Reduktion. Zwei Studien gaben die Krankenhausverweildauer an, diese verkürzte sich unter kombinierter Therapie um etwa 2 Tage.

10 Studien machten Angaben zu Nebenwirkungen. Diese waren moderat ausgeprägt und zwischen beiden Gruppen nicht signifikant verschieden $(p=0,82)$. Der Funnel-Plot-Test bzgl. Thorax-CT zeigte Hinweise auf einen leichten Publication-Bias-Effekt.

Das Review schlussfolgert, dass chinesische Medizin bei der Behandlung von COVID-19 vorteilhaft sein könnte („may be benificial“"), dass jedoch wegen der methodischen Schwächen weitere rigoros durchgeführte Studien nötig sind, um die Ergebnisse zu bestätigen.

\section{Kommentar}

Die Arbeit stellt das erste Review einschließlich Metaanalyse zur chinesischen Arzneitherapie bei COVID-19 dar, das strenge Kriterien anlegt. Die Autoren haben dieses in unglaublich kurzer Zeit erstellt: Die Datenbankrecherche lief bis zum 21.06., die Onlineveröffentlichung der Arbeit datiert vom 02.07.2020. Allerdings ist die statistische Auswertung zu bemängeln (s. unten). Auch sind einige methodische Details meines Erachtens unzureichend beschrieben, z. B. nach welchen Kriterien wurde eine Verbesserung des Thorax-CT-Befundes beurteilt? Die Tatsache, dass der Thorax-CT-Befund als Hauptzielgröße ausgewählt wurde, obwohl dieser Parameter in einem Teil der Studien gar nicht erhoben wurde, ist zu hinterfragen.

Das Review zeigt respektable Ergebnisse zugunsten einer $\mathrm{Zu}$ satzbehandlung mit chinesischer Arzneitherapie auf, die für fast alle Zielparameter so konsistent ausfallen, dass es fast zu schön, um wahr zu sein, scheint. Diese Ergebnisse können jedoch nicht über erhebliche methodische Schwächen der eingeschlossenen Studien hinwegtäuschen. Der Randomisierungsmodus bleibt überwiegend unklar. Die Verblindung der Beurteiler ist nur in einer Studie gewährleistet, in einer verneint, in allen anderen bleibt sie offen. Nicht besser ist es um die Verblindung der Behandler bestellt. Somit könnte die Beurteilung von Symptomen oder des klinischen Verlaufs subjektiven Maßstäben unterlegen haben. Bei der Vielzahl der getesteten Zielparameter wurde eine statistische Korrektur für multiples Testen vernachlässigt, sodass bei entsprechender Adjustierung möglicherweise nicht alle Signifikanzen Bestand hätten.
Der härteste Endpunkt, die Sterberate, fällt nicht signifikant verschieden aus. Man fragt sich, warum dieser wichtige und leicht zu erhebende Parameter nur in 4 der 18 Studien mitgeteilt wurde. Andere objektive Parameter, wie Virusload, CRP und Krankenhausverweildauer, erweisen sich immerhin als signifikant. Insgesamt gewinnt man den Eindruck, dass angesichts der Fülle der erhobenen Parameter, die zugunsten der Kombinationsbehandlung ausfallen, vieles für einen positiven Einfluss der chinesischen Medizin spricht. Dabei lag die Therapiedauer in 5 Studien nur zwischen 5 und 7 Tagen, ein Zeitraum, in dem das Potenzial der chinesischen Medizin vermutlich noch nicht voll ausgeschöpft gewesen sein dürfte.

Eine international vorzeigbare Evidenz, die eine Aufnahme der chinesischen Arzneitherapie in internationale Leitlinien rechtfertigen würde, wird mit diesen Ergebnissen nicht generiert. Zum großen Teil liegen die methodischen Schwächen sicherlich an dem Zeitdruck, der erst einmal ein rasches Handeln zum Wohle der erkrankten Patienten erforderte und wenig Zeit für Forschungsplanung ließ. Auch westliche Studien, die mittlerweile beendet wurden, weisen oft keinen hohen Standard bzgl. Randomisierung oder Verblindung auf. Da inzwischen die Erkrankungsrate an COVID-19 in China auf ein Minimum zurückgegangen ist, sind weitere Studien, die methodisch besser angelegt sind, aus dieser Region wohl nicht mehr zu erwarten.

Korrespondenzadresse

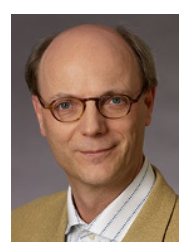

Dr. Axel Wiebrecht

Offenbacher Str. 5, 14197 Berlin, Deutschland

axel.wiebrecht@gmx.de

Interessenkonflikt. A. Wiebrecht gibt an, dass kein Interessenkonflikt besteht. 